American Journal of Economics and Business Administration 4 (1): 47-58, 2012

ISSN 1945-5488

(C) 2012 Science Publications

\title{
Gravitational Force of Community Events: A New Method of Evaluating Value of Local Events
}

\author{
${ }^{1}$ Nathanael L. Adams and ${ }^{2}$ Lynn L. Adams \\ ${ }^{1}$ College of Business and Management, \\ Cardinal Stritch University, 6801 N. Yates Road, Milwaukee, WI, 53217, USA \\ ${ }^{2}$ Department of the Finance and Economics, Woodbury School of Business, \\ Utah Valley University, West University Parkway, Orem, UT, 84058, USA
}

\begin{abstract}
Problem statement: This proposed "Gravitational Force" model was created to give communities a way to measure how effectively local events attract participants and visitors from a distance. Additionally, this study shows how to quantify the economic effect of an event on the local tax base and the entire community. This model can be used for all kinds of events and can help assess different kinds of events in relationship to each other. After the US and world economy faltered in 2008 many governmental bodies had to find ways to cut budgets. Community events are considered discretionary, but that can have a large effect on a local economy, both in terms of actual cash flows and in branding the community. Conclusion/Recommendations: This study presents a new way to measure local events and attractions that takes into account how far out an event tends to attract participants and to what degree by using packages available to $\mathrm{R}$ and a new set of ratios based on the number and duration of hotel occupants. This study will suggest a framework for local leaders to assess priorities when using these methods.
\end{abstract}

Key words: Gravitational, force, community events, local events

\section{INTRODUCTION}

In the wake of the economic turn down after 2008 , municipal, county and state governments in the United States and elsewhere in the world have struggled with reduced tax revenues. Discretionary funds for these government bodies have been very tight and so a need exists to carefully evaluate the best way to spend public monies. Since some public needs are so critical, such as public safety, utilities and the like, that the main cuts in many budgets have been in such areas as community events and other budget items that are seen as less critical. Since community events are on the "chopping block" in so many cases the authors of this study thought that a methodology to evaluate the economic value of events would be of great value to many governing bodies.

One very important measure of any community event is how far away people are drawn to an event. If people come to an event from a distance that is not within a comfortable distance to travel within a day they will tend to stay at a motel, thus greatly increasing the potential economic impact of the event. Both of the authors of this article grew up in a small community (Heber, Utah) that is close to the heavily populated Wasatch Front. Many of the local events and attractions in the Heber Valley draw heavily from the Wasatch Front and the result is that the economic impact outside the money paid to the particular attraction is minimal, usually only consisting of some food purchases and perhaps some gas for their vehicle. However, if a visitor stays longer than a day the amount of money spent in the local economy greatly increases as that person spends on such things as a motel/hotel accommodations, extra meals, extra shopping, rental cars, so, one important measure of any event is how far away on average that the event attracts participants. This study develops a methodology to measure the attraction (gravity) of events and then suggests a way to incorporate that knowledge into a decision making process.

To test the methodology that the authors have been working with, two events (one is a tourist attraction in its own right) in Wasatch County were chosen. The first was the Heber Valley Railroad (HVRR), which had already been the subject of a marketing study by the authors (Adams and Adams, 2006) and the second was a specific event at the Soldier Hollow Ski Resort (SOHO), a Junior Olympics cross country ski event held on January 28, 2011. The Heber Valley Railroad evaluation was for an entire year, while the Soldier Hollow event was a week long race for juniors.

Corresponding Author: Nathanael L. Adams, College of Business and Management, Cardinal Stritch University, 6801 N. Yates Road, Milwaukee, WI 53217, USA Tel: 608-239-6990 

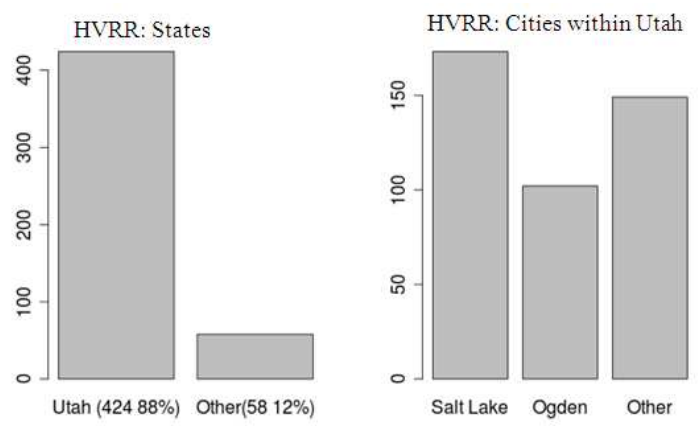

Fig. 1: HVRR customer histogram

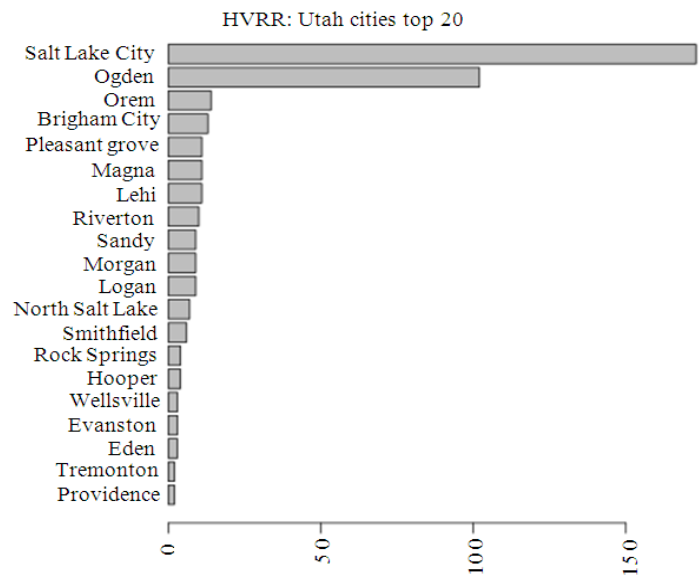

Fig. 2: HVRR Customers, Utah cities top 20

Problem: The once flourishing US economy has sputtered, leaving municipalities struggling to decide how to spend an ever-smaller pool of money. This study does not focus on debt reduction, or future investments. Instead it tries to address a question about current and past community events and their potential to bring in revenue for the community. To put the problem explicitly, what events should continue to get money and what events should not? Some well-established methodologies for determining profitability already exist, fitting community vision and values, the prestige of the event and more.

What makes this study unique is the use of "gravity" or how far out an event attracts participants, as a way of measuring the importance and potential profits for the community. Gravity can be defined as the "attraction" force between two objects (Halladay and Resnick, 1974) and can be seen every day as objects fall to the ground. Using a roughly similar definition the analysis looks at the "gravitational" pull of an event to determine potential value. What makes this analysis different from the force that keeps the celestial bodies in perpetual motion is gravity for any event is unique and different from other events, making event's gravity a variable, which can change over time and not a constant. Instead of the attraction that exists between two masses, individual events or attractions have a unique attraction for participants. The two factors affecting the gravitational pull of an event are the number of people attending and the distance traveled to get there. Using distance and number of attendants, municipalities can understand which events draw from a larger area and thus to put extra funding into.

Background: The idea for this article came from a speaker at Pepperdine University, who had the idea of trying to show the gravitational pull of particular cities and events. At the time it was a theoretical idea and was an aside given during a talk. Unfortunately the authors do not remember who that person was, but the seed was planted and the idea grew. After about six years of thought the idea was resurrected with a blog about creating maps using the great circle route between points and finding the distances (Lamigueiro, 2011).

What the blog showed was how to take data like cities, longitude and latitude, then using $\mathrm{R}$ to make a graph of the data. The example on the blog was the different flights on American Airlines and the frequency of the route (Lamigueiro, 2011). The idea of creating an equation is one thing, but the visual display of showing how dramatically the number of people coming and going was also needed. $\mathrm{R}$ allowed the authors the ability to show graphically and numerically how much gravity an event has. This idea coupled with the current economic situation where many municipalities are asking themselves how to spend less and less, while maintaining and supporting profitable events. To this end, a series of quantitative evaluations and graphs were created to explore the gravitational draw of two very different community draws (Fig. 1-10).

\section{MATERIALS AND METHODS}

This materials and methods section is broken into three major parts; the first is a discussion of the assumptions and limitations of the latitude and longitude method of determining distance. The " $R$ " section, which then follows describes the assumptions, tests and results in the creation of the code for the $\mathrm{R}$ program developed for the analysis. The describes how the data derived in the $\mathrm{R}$ program were cleaned up.

Latitude and longitude assumptions/limitations: The model hinges upon deciding on a reasonable choice of what constitutes a local visitor versus the "Out-of-Towner." Additionally the number of hours and distance traveled divided these two groups of visitors turned out to be a challenging question. 
Am. J. of Economics and Business Administration 4 (1): 47-58, 2012
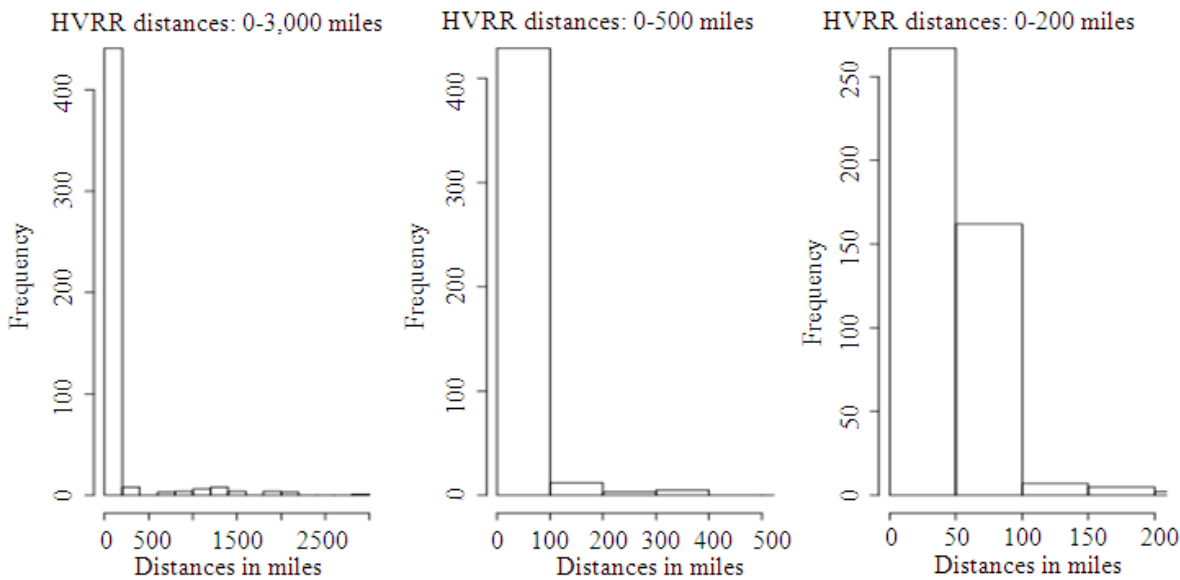

Fig. 3: HVRR customer histograms

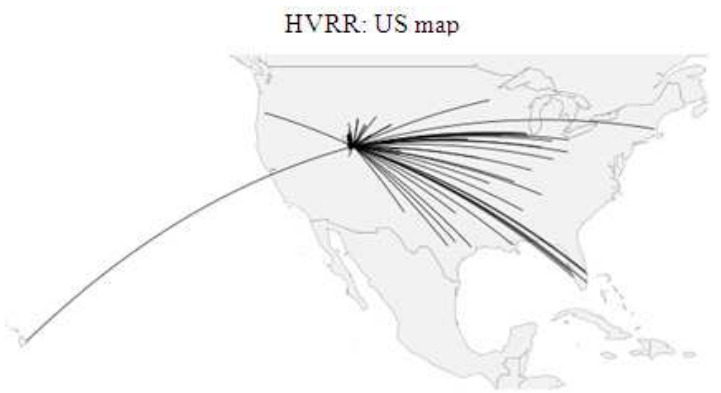

Fig. 4: HVRR great circle customer distribution

For example on a flat plane the distance from point $\mathrm{A}$ to point $\mathrm{B}$ is simple. In the complex highway system where there might not be a direct route and there are rivers, lakes, mountains, canyons and other natural obstacles, there is no direct route. Theoretically the ideal solution would be to get the address of every visitor then get a map with the driving time and distance from MapQuest.

Three major problems exist with the MapQuest solution, first the programming needed to get all of that information would make the model bulky and difficult to use. Second is the problem of getting the address of every visitor to an event. In the hyperprivacy sensitive world people are not willing to give out their address to a stranger. Additionally, collecting and aggregating such information is difficult even for technology savvy municipalities. The third problem is what if the visitor is flying? This complication makes the analysis even more problematic. One solution is to use city names, states and zip code to find the latitude and longitude coordinates and then ignore both terrain and mode of transportation.

Latitude and longitude coordinates take minimal information, city, state, country or zip code; all of which people are more willing to give. Ignoring geographic features remove all the natural barriers to travel by using 'as-the-crow-flies' distances, giving the absolute minimal distance to travel. Using the minimal distance traveled consistently the lowest gravity weight can be given to each visitor and each event; and will be consistent throughout different analysis. Deciding upon using the latitude and longitude method led to the question of which distance equation to use in $\mathrm{R}$.

R: $\mathrm{R}$ is an open source statistical program used for a wide range of statistical applications and can be found at www.r-cran.org (Team, 2011). Because R is open source contributors from all branches of academia can contribute packages, or additional commands and data to the base program. The two packages used for this analysis are the Geosphere (Hijmans et al., 2011) and Maps (Becker, et al., 2011) packages. The Maps package includes the maps needed to graph the data and a database of world cities with a population greater than 40,000. The Maps database of cities was used to generate a simulation where the different formulas for distance were measured and tested for speed and accuracy.

The formulas used to determine the distance between the two objects are found in the Geosphere package and include Haversine, Vincenty sphere and Vincenty elliptical. Both the Haversine and Vincent Y sphere formula assumes a spherical earth with a radius of 6,378,137 meters using a great-circledistance assuming no terrain obstacles, mountains, rivers, (Hijmans et al., 2011). The third formula is the Vincenty elliptical formula, which assumes the earth is elliptical, while utilizing the great-circle-distance for distance measurements. The question for the researchers was which formula to use?

To determine which formula to use a test was created to determine the accuracy of each formula and the speed for each one. The Maps database of 
1,005 United States cities was used with a starting point of New York and then each sum distance was found using each formula and timed. The time is the processor time using an AMD 64 (2.1G dual processor, with 4 Gig of Ram, running Mint10 Linux in RStudio; times will vary based on the hardware, number of processes running in background, OS and other factors. The data were converted to miles during the summing process.

From the results the Haversine and Vincent $\mathrm{Y}$ spheres equations are exactly the same both in distance and processing time. The Vincenty ellipsoid shows a significantly more miles than the other two $(1,755,397)$ and the processing time is double the time as the others, but at 2.51 seconds (Table 1). For 1,005 data points the extra second is worth the extra precision as the average distance error between the Haversine and the Vincenty ellipsoid is 1.08 miles per data point, meaning the sphere formulas are on an average 1 mile short of the more accurate ellipsoid formula. The formula used then is the Vincenty ellipsoid formula for the distance measurements. For additional information Appendix A for the $\mathrm{R}$ code used for the test.

\section{Appendix A:}

\section{Distance formula evaluation code in $\mathbf{R}$ :}

Require (geosphere)

Require (maps)

Data (us. cities)

\#Setting up the data, 'ny' is the long, lat for New York City, 'all' is a matrix of all the cities

\# available in the geosphere package (1005), with the long. and lat. data.

ny<-c(-118.41, 34.11)

all<-matrix(data=c(us.cities\$long, us.cities\$lat), $\mathrm{ncol}=2$ )

\#Summing the distance between NY and all the other cities in the US (1005 of them)

\#by so making the error is compounded with each additional city

have $<-\operatorname{sum}(\operatorname{distm}($ ny, all, fun=distHaversine $)$ )

have.time<-proc.time()

v.Sphere<-sum(distm(ny,

fun=distVincentySphere))

v.Sphere.time<-proc.time()

v.Ellip<-sum (distm(ny,

fun=distVincentyEllipsoid))

v.Ellip.time<-proc.time()

have.time; v.sphere.time; v.ellip.time;

proc.time<-c $(1.350,1.350,2.510)$

row.names<-c('Haversine','Vincenty.Sphere',

'Vincenty.Ellipsoid')

ny.all<-rbind(hav, v.sphere, v.ellip); ny.all<cbind(ny.all, proc.time)

rownames(ny.all)<-row.names; colnames(ny.all)<c('Sum Distance', 'Processor Time'); ny.all
\#Determining the difference between the various models available in the geosphere package

\#Meters were converted into miles, the largest difference between the models was approximately \#1090 miles, or 1.085326 miles per city of difference, this is considerable

hav.v.ellp<-(v.ellip-hav)*0.000621371192

hav.v.sphere<-abs(hav-v.sphere)*0.000621371192

hav.v.ellp; hav.v.sphere

diff<-rbind(hav.v.ellp, hav.v.sphere)

rownames(diff) <-c('Haversine-

Vincenty.Ellipsoid','Haversine-Vincenty.Sphere')

colnames(diff)<-'Distance (miles)'; diff

\#what is the average error

hav.v.ellp/1005

\# At the end the Vincenty. Ellipsoid was used as the method for determining the distance as it was

\# the most precise by an average margin of 1.0853

miles per city, this is a significant margin of

\# error when many cities are being analyzed and the extra computing time in negligible

The second group of code found in appendix B is the code used to generate the analysis for each of the organizations, the Heber Valley Railroad (HVRR) and Soldier Hollow (SoHo). Each set of code is broken into three major parts; the first portions of the analysis include descriptive statistics such as mean, mode, median, standard deviation and Pearson's skews. The reason Pearson skewness is used because of the simplicity in understanding what it means. The Pearson skewness ranges from -3 to 3 , where -3 is negatively skewed, 3 are positively skewed and 0 is symmetrical. There are other methods of determining the symmetry of a distribution, but the assumption is that those who will be making the decisions will not understand nor appreciate complex formulas when a simple one is available.

The second part of the analysis code is the exploratory graphs showing important information all, for each organization. These are a combination of frequency tables and histograms to visually describe the data presented (for example Fig. 1). The distance histogram and maps use the Vincenty ellipsoid formula to maximize accuracy and to be consistent with the analysis. The final part of the code is the code needed to create the various maps for the analysis of distance.

Table 1 Results of formula test

\begin{tabular}{lll}
\hline & Sum distance & Processor time $(\mathrm{sec})$ \\
\hline Haversine & $2,327,309,913$ & 1.35 \\
Vincenty. Sphere & $2,327,309,913$ & 1.35 \\
Vincenty. Ellipsoid & $2,329,065,310$ & 2.51 \\
\hline
\end{tabular}


Appendix B:

Code for HVRR and SoHo analysis in R:

require(geosphere)

require(maps)

\#HVRR Analysis

\#Step 1: basic Stats. Summaries, Histograms, bar charts

\#reading the file in

hvrr<-read.table(file.chooses(), header=TRUE)

\#summary stats

summary(hvrr)

\#histograms

$\operatorname{par}(\mathrm{mfrow}=\mathrm{c}(1,2))$

label.1<-c('Utah (424 88\%)', 'Other(58 12\%)')

state<-c $(424,58)$

barplot(state, names.arg=label.1, main='HVRR:

States', col='blue')

label.2<-c('Salt Lake City', 'Ogden', 'Other')

cities<-c(173, 102, 149)

barplot(cities, names.arg=label.2, main='HVRR:

Cities Within Utah', col='blue')

$\operatorname{par}($ las $=2, \operatorname{mar}=\mathrm{c}(5,12,4,2), \mathrm{mfrow}=\mathrm{c}(1,1))$

city. $1<$-sort(table(hvrr\$city))

city. $1<$-tail(city.1, $n=20)$

barplot(city.1, col='blue', hor=TRUE, main='HVRR:

Utah Cities Top 20')

$\operatorname{par}($ las $=0, \operatorname{mar}=\mathrm{c}(5,4,4,2))$

\#distance analysis

heber<-c(-111.33259, 40.511413)

data $<-$ matrix $($ data $=c($ hvrr\$long, hvrr\$lat $)$, nrow $=482$, ncol=2)

ut $<$-subset(hvrr, subset=(st=='UT'))

data.ut<-matrix (data=c(ut\$long, ut $\$$ lat $),$ nrow $=424$, $\mathrm{ncol}=2$ )

dist $<-($ distm (heber,

fun=distVincentyEllipsoid)*0.000621371192)

dist.rr $<$-matrix (dist, nrow $=482$, ncol $=1$ )

hvrr<-cbind(hvrr, dist.rr)

\#histograms of various shapes and zooms

summary(dist.rr)

$\operatorname{par}(\operatorname{mfrow}=\mathrm{c}(1,3))$

hist(dist.rr, breaks=12, main='HVRR Distances: 03,000 miles', xlab='Distance in Miles', col='blue')

hist(dist.rr, breaks=24, main='HVRR Distances: 0500 miles', $x l a b=$ 'Distance in Miles', $x \lim =c(0,500)$, col='blue')

hist(dist.rr, breaks=50, main='HVRR Distances: 0200 miles', $x l a b=$ 'Distance in Miles', $x \lim =c(0,200)$, col='blue')

$\operatorname{par}(\operatorname{mfrow}=\mathrm{c}(1,1))$

\#mapping it out

\#US

map("world", col="\#f2f2f2", fill=TRUE, bg="white",

$1 w d=0.25, x \lim =c(-158,-65), y \lim =c(15,50))$

title(main='HVRR: US Map')

for(i in $1: \operatorname{dim}($ data $)[1])\{$ inter <- gcIntermediate(heber, data[i, 1:2], $\mathrm{n}=482$, addStartEnd=TRUE)

lines(inter, col="blue")

\}

\#Zoomed into Utah

$\operatorname{par}(\operatorname{mfrow}=\mathrm{c}(1,1), \operatorname{mar}=\mathrm{c}(5,4,4,2))$

map("state", col="\#f2f2f2", fill=TRUE, bg="white", $1 w d=0.25, x \lim =c(-115,-108), y \lim =c(37,42))$

title(main='HVRR: Utah')

for(i in 1:dim(data.ut)[1])

inter <- gcIntermediate(heber, data.ut[i, 1:2], $n=424$, addStartEnd=TRUE)

lines(inter, col="blue")

\}

\#Wasatch Front

map("state", col="\#f2f2f2", fill=TRUE, bg="white", lwd=0.25, $\operatorname{xlim}=c(-112.5,-111), \operatorname{ylim}=c(40,42))$

title(main='HVRR: Utah- Wasatch Front')

for(i in $1: \operatorname{dim}$ (data.ut)[1])

inter <- gcIntermediate(heber, data.ut[i, 1:2], n=424, addStartEnd=TRUE)

lines(inter, col="blue")

\}

$\operatorname{par}(\mathrm{mfrow}=\mathrm{c}(1,1))$

\#Soldier Hallow Analysis

soho<-read.csv(file.choose(), header=TRUE)

summary(soho)

table.city<-sort(table(soho\$city), decreasing=TRUE)

table.st<-sort(table(soho\$state), decreasing=TRUE)

$\operatorname{par}(\operatorname{mar}=\mathrm{c}(5,11,4,2)$, las $=2)$

barplot (table. City, main='SoHo: Cities', horiz=TRUE, col='red')

$\operatorname{Par}(\operatorname{mar}=\mathrm{c}(5,4,4,2)$, las $=2)$

barplot (table. St, main='SoHo: States', horiz=TRUE, col='red')

Heber<-c $(-111.33259,40.511413)$

soho.data<-matrix (data $=c($ soho\$long, soho\$lat),

nrow $=373$, ncol=2)

Soho. UT<-subset $\left(\right.$ Soho, subset $=\left(\right.$ state $\left.\left.=={ }^{\prime} U T '\right)\right)$

soho.data.ut<-matrix (data $=\mathrm{c}$ (soho.ut\$long,

soho.ut $\$$ lat $)$, nrow $=29$, ncol $=2$ )

soho.dist<-(distm(heber, soho.data,

fun=distVincentyEllipsoid $) * 0.000621371192$ )

soho.dist.ut<-(distm(heber, soho.data.ut,

fun=distVincentyEllipsoid)*0.000621371192)

dist.soho<-matrix(soho.dist, nrow=373, ncol=1)

dist.soho.ut<-matrix(soho.dist.ut, nrow=29, ncol=1)

Summary (Dist. SOHO)

SD (Dist. SOHO)

P. Skew. Soho<-(3* (mean (Dist. Soho) -median (Dist. SOHO)) ) /SD (Dist. SOHO)

Heist (Dist. Soho, main='SoHo: Distance Histogram', col='red')

Heist (Dist. SOHO. UT, main='SoHo: Distance Histogram Utah', breaks=20, col='red')

\#mapping it out

\#US 
map("state", col="\#f2f2f2", fill=TRUE, bg="white", $1 w d=0.25)$

Title (main='SoHo: US Map')

Four (I in 1: dim (SOHO. Data) [1]) \{

Inter <- gcIntermediate (Heber, Soho. Data [I, 1:2], $\mathrm{n}=373$, addStartEnd=TRUE)

Lines (inter, col="red")

\}

\#Zoomed into West

Par $($ mfrow $=\mathrm{c}(1,2)$, mar $=\mathrm{c}(5,4,4,2))$

map("state", col="\#f2f2f2", fill=TRUE, bg="white", $1 w d=0.25, x \lim =c(-125,-103), y \lim =c(30,50))$

Title (main='SoHo: Western Region')

for(i in 1:dim(soho.data)[1])\{

Inter <- gcIntermediate (Heber, Soho. Data [I, 1:2], $\mathrm{n}=373$, addStartEnd $=$ TRUE)

Lines (inter, col="red")

\}

\#Utah

map("state", col="\#f2f2f2", fill=TRUE, bg="white", lwd=0.25, $x \lim =c(-112.1,-111), \operatorname{ylim}=c(40,42))$

Title (main='SoHo: Utah')

for(i in 1:dim(soho.data.ut)[1])\{

Inter <- gcIntermediate (Heber, Soho. Data. UT [I, 1:2], $\mathrm{n}=29$, addStartEnd=TRUE)

Lines (inter, col="red")

\}

Par $($ mfrow $=\mathrm{c}(1,1))$

Data cleanup: The data was cleaned up and zip codes were used as the primary means of finding the longitude and latitude for each point. The zip code offers the advantage of being more precise. Major cities have more than one zip code within them by using the zip codes a more precise set of distances can be found. For the HVRR zip codes were provided and so this method was used. For the SOHO data did not have the zip codes so the generalized one city, one zip code, one latitude and longitude was used; knowing there is a lack of precision within the model compared to the HVRR.

\section{RESULTS AND DISCUSSION}

What follows is a discussion of how the Heber Valley Railroad and Soldier Hollow Junior Olympics event were analyzed and the raw data presented.

Heber valley railroad: The analysis of the HVRR starts with understanding the distance data and the various levels contained therewith. The distance analysis starts with the frequency of states and one conclusion was clear from the data, Utah is number one. At $88 \%$ Utah has the highest number (Fig. 1); the next closest was Wyoming with 16, Florida at 6 and Colorado and Texas in 4 . From that point the data drops off quickly making it hard to distinguish with
Utah so high. Having examined the states the next phase was the cities within Utah.

Of the cities within Utah the two biggest are Salt Lake and Ogden, the other group are all the other cities that, like the state data drops 1-4 for each city. The top 20 cities graph (Fig. 2) shows how quickly the frequency of cities drops off after Salt Lake City and Ogden. From the frequency of states and city, the main source of customers come from Utah and mainly lives in Salt Lake City and Ogden; both of which are within a one-day's drive to HVRR, making it an ideal one day activity.

From this point a series of histograms were created based on the distance using the Vincenty ellipsoid formula. The histograms start out with all of the data points to show how positively skewed the data is and a distance range of 0-3,000 miles (Fig. 3). The second histogram zooms into the data with a range of 0-500 miles and an interval width of 100 miles. The final histogram has a range of 0-200 miles and an interval width of 50 miles, showing clearly how the majority of customers for the HVRR are within 100 miles of the railroad.

The next series of graphs are the maps with a great-circle-curve from the origin (Heber City, Utah) to the participant's location (Fig. 4 and 5). Figure 4 is a map of the United States with Hawaii, showing a considerable number of participants who come from various areas of the United States. Some visitors are particular groups from Florida, Wyoming, Texas and the Midwest. The second set of maps removes all other participants except for those from Utah (Fig. 5) and then zooms into what is termed the Wasatch Front, or the metropolitan areas of Salt Lake City, Ogden and Provo.

The maps and histograms demonstrate how positively skewed the HVRR distance data is and how the majority of the participants are coming from within 100 miles of HVRR. This is important information for HVRR as they need to know how to advertise and where. Yes participants are coming from all over the United States, but the number is so low, the return on investment if marketing were to be conducted nationally would not be adequate. At this point it should be noted that the data for HVRR is a sample of all the participants from HVRR during an entire year, not a single event. So for the HVRR the majority of participants come from within 100 miles making it a day trip activity, not a multi-day activity or event.

Soldier hollow: The SOHO analysis began by separating the states of participants, which shows more variety compared to HVRR (Fig. 4). Utah is 4th on the list compared to HVRR where it was first. This difference is significant because the majority of participants is coming from a greater distance than a 
simple day trip. Junior Olympics participants have to stay in or near Heber City in order to participate. A higher percentage of overnight visitors helps the economy of Heber City and nearby towns as restaurants and hotels are required, thus significantly more money spent by these participants as opposed to HVRR customers who may stop at a restaurant, but not likely stay at a hotel. The next graph for SOHO shows the wide range of cities the participants are coming from (Fig. 7). From a marketing perspective this is a bit problematic as there is no central concentrated location to focus marketing. But, because cross-country skiing is relatively specialized business segment marketing can be focused in a few publications and organizations.

The next SoHo histogram below (Fig. 8) shows how much more normally distributed the SoHo data is compared to the positively skewed data of HVRR. Because participants are coming from specific areas and several from each location, the data are more evenly distributed, as compared to the large mass of HVRR.

The next graphs are the maps for the SOHO group, where the first one is in the United States (Fig. 9), then the Western States and Utah (Fig. 10). The variety of locations from which participants are coming to SoHo for this event is limited to the Western region of the United States. One reason may be due to monetary restrictions caused by the downturn in the economy. Remember this is only one event of many during the year at SOHO, as compared to the aggregate for HVRR over several years.

The analysis of the map graphs shows how participants are coming from greater distances, while participants are not concentrated from Utah. This dispersion is good for Heber City, as these participants have to stay at least one night, so more money is spent in the Heber Valley on top of the cost of the event. The distance summary statistics reaffirm the conclusion that SOHO has more people coming from greater distances, versus HVRR that is more of a day trip (Table 2).

Limitations: This study has already explained the limitations by using zip codes and distance formulas; which are that the distances used for this model do not account for flying versus driving or any other differences in modes of transportation and that different modes of transportation will not only have longer distances than those used in this model, but the time of travel could be significantly different. Another limitation is the reality that having participants in events stay more than a day does not guarantee that they will use community motels or hotels. When using this model, each entity will have to estimate the additional economic impact of having people stay longer than a day. Wasatch County has conducted just such a study (Wasatch County Visitors Survey, 2010, Appendix C), so the results of this model and rubric will be relatively easy to apply in that county. This gravitational model is just one factor of many that an entity could or should evaluate when making strategic decisions by using a rubric.

What follows in this Results section is a discussion of how the raw data from the $\mathrm{R}$ program was used to create an events rubric for governing bodies of community events and how to use the rubric.

Table 2: Distance summary statistics

\begin{tabular}{lrr}
\hline & HVRR & SoHo \\
\hline Min & 10.49 & 0.00 \\
Median & 39.23 & 284.40 \\
Mean & 141.90 & 299.40 \\
Max & 2945.67 & 704.20 \\
Skewness & 0.86 & 0.31 \\
\hline
\end{tabular}

HVRR: Utah-Wasatch Front
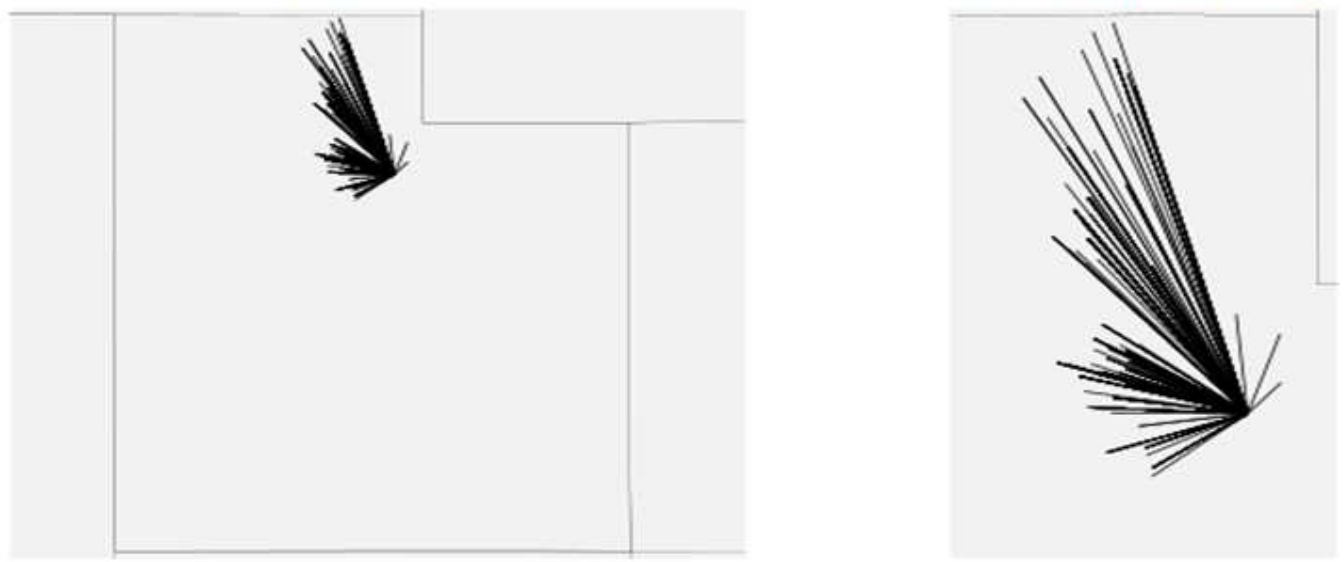

Fig. 5: HVRR great circle histogram for Utah 
Appendix C: The following table shows a comparison between segments of the population with respect to their average spending per day during their stay in Heber Valley, Utah, USA. The table has been copied from page 8 of the Wasatch County Visitors Survey Report Prepared for Wasatch County Tourism and Economic Development Office, dated November 1, 2010. The full 84 page report and questions about the report can be acquired and answered through Ryan Starks, Director of Tourism and Economic Development for Wasatch County (phone 435-6543666, email ryanstarks@gohebervalley.com):

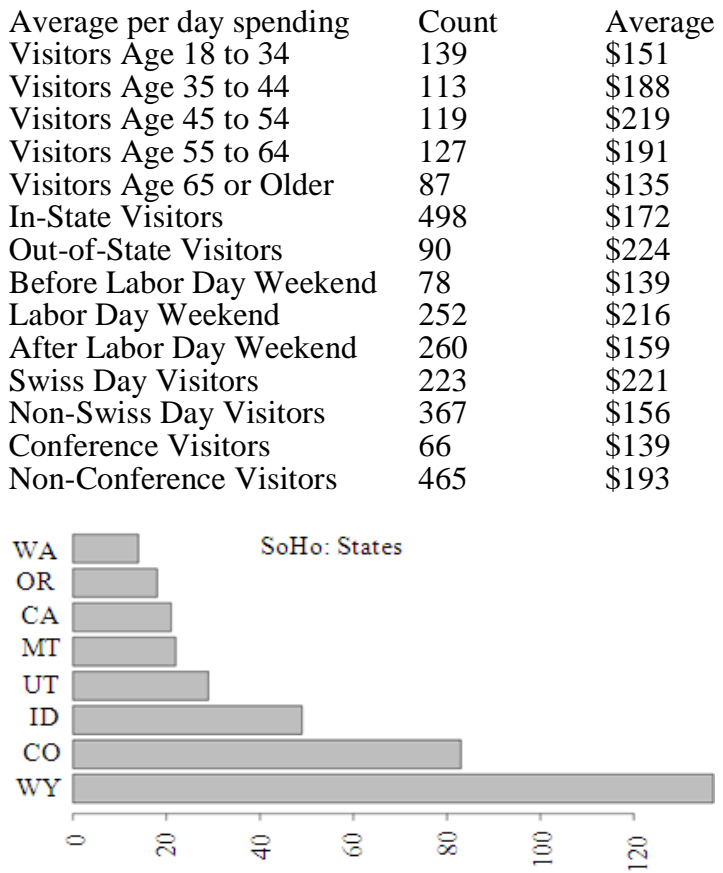

Fig. 6: SOHO Jr. Olympics histogram by state
A possible events rubric: When events are evaluated, especially events that have been around for some time and have become somewhat "sacred" to the community and leaders, people tend to look first at the notoriety or how fashionable the event is. In short, events take on a persona and are also marketed to have an appealing image, thus some events become very emotionally tied to the community and/or the organizers. The authors felt that to maintain some semblance of objectivity an event must first be evaluated based on a measurable economic performance rubric.

The first discriminator is whether or not a specific event pays its own way. Some events or attractions generate enough cash flow to pay any community (government or other) costs. In this study the HVRR has historically fit into the category of paying its own freight, except for some loans from Heber City, Wasatch County governments and some grants. The Junior Olympic races at SOHO however fit into the category of being a significant cost to the venue. The reason that cross country ski events are being held in the post 2002 Olympics era is that $\mathrm{SOHO}$ is also a nonprofit sports foundation whose stated purpose is to produce competitive cross country skiers both at home and in the United States in general. SOHO, then, has to balance the number and cost of events that fit its mission of producing great skiers with those events and activities that produce the cash flow that keeps the venue in the black.

Once an event is separated into one of the two aforementioned classes, those events that sustain themselves and those that come at a cost, then the next step is to evaluate those cash flows or costs.

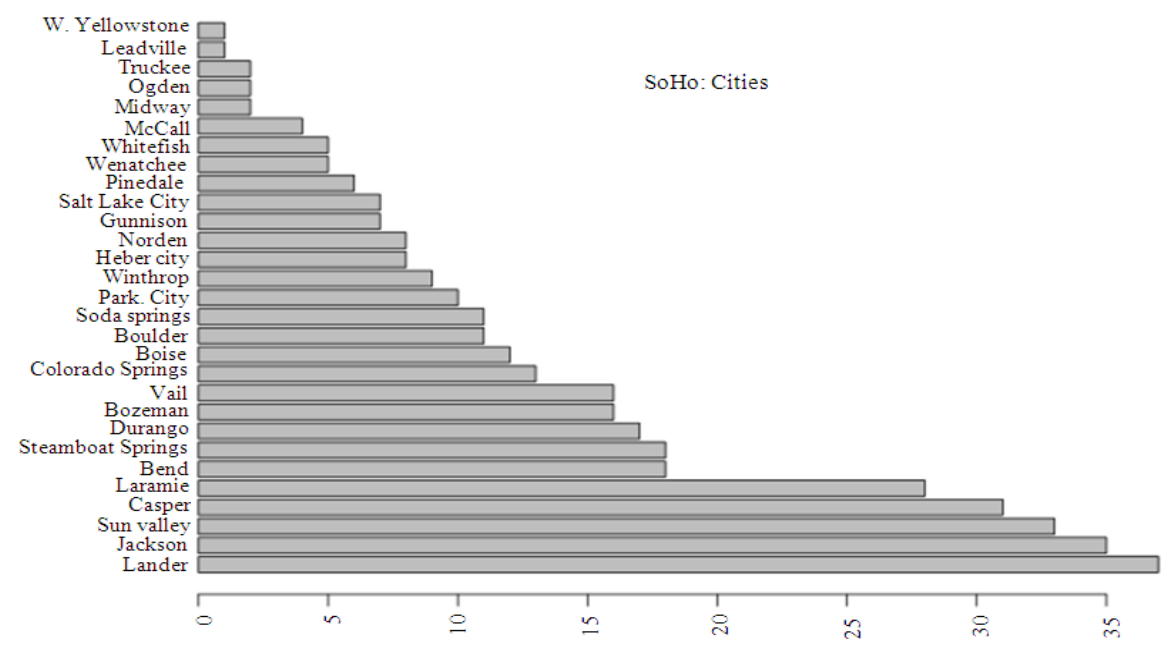

Fig. 7: SOHO Jr. Olympics cities histogram 


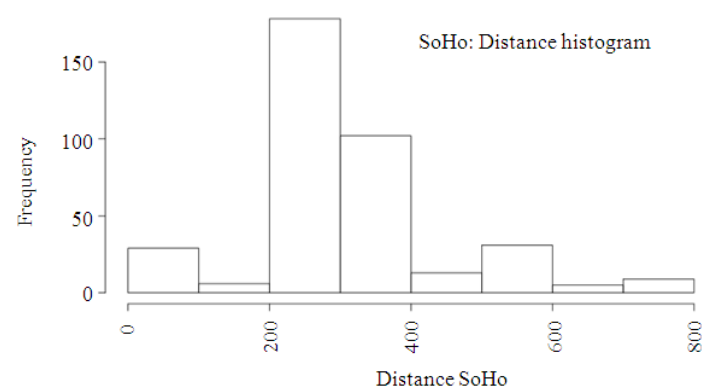

Fig. 8: SOHO distance distribution histogram

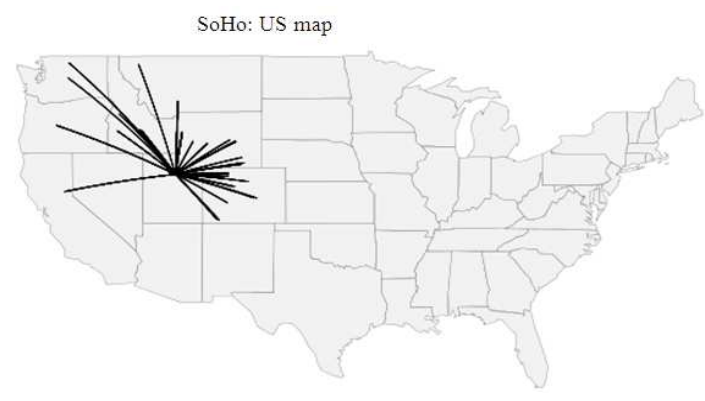

Fig. 9: Great Circle Histogram, SOHO

Because SoHo is becoming calendar limited due to the popularity of the venue year round and the fact that SoHo has a small full time staff, the venue has to really look hard at both sustainable events and legacy mission events that bring significant cost to the venue. A large percentage of the new and total revenue is from specialized sports events, weddings and the venue's annual Sheep Dog Classic event. The Soldier Hollow Legacy Foundation Board and the General Manager, Howard Petersen, have to do a fine balancing act between legacy events and other community events that are not yet sustainable and activities that are profitable. And, due to the limited staff, the events are being looked at on a profit per day basis.

The SOHO example shows how different entities in the same community will look at a specific event in a totally different manner. The JO races used in this study come at a cost to the venue, yet those events fill basically every motel in the valley, spilling over into other communities. Soldier Hollow spent \$22, 023.63 on competition events in $2010-11$ and is budgeting $\$ 86,750$ for 2011-12 (Soldier Hollow Financials, shown in Spring 2011 Soldier Hollow Legacy Foundation Board meeting minutes, available by email through Howard Peterson at howard.peterson@soldierhollow.com). Wasatch County reaps a windfall of the hotel and other taxes and the total money spent in the larger community is significant. Businesses, especially the motels, see full occupancy rates during JO races and since cross- country ski competitors can eat 5000 or more calories a day the eateries and grocery stores see additional sales.

Shortly after the Olympics in 2002 the Wasatch Chamber of Commerce estimated that people who stay overnight or longer spend roughly $\$ 100$ a day per person, while those who visit for an event for just the day spend about $\$ 20$ per person. The numbers were estimated in part due to the disparity between snow mobiles, who were almost exclusively day only visitors and whom the community was lucky if they bought gas and ate in the area and downhill skiers who tended to stay a week or a weekend. These numbers were just estimates. The recent Wasatch County Visitors Survey (Appendix C) showed that overnight visitors spent between $\$ 135$ and $\$ 224$. Assuming an average of $\$ 180$ per day per individual that stays in a motel, the JO events could possibly bring in roughly $\$ 540,000$ into this small community in six days. Unfortunately the survey did not show what visitors will spend if they do not stay overnight.

Direct revenues can thus be measured and as shown below can be broken down into the revenues provided by multi-day visitors and those who only stay during the day (Table 3). Several ratios are shown, including the Hotel Ratio, which measures the ratio of visitors of more than one day versus day only visitors. The higher the number the more an event draws from significantly outside the local area. The Hotel Duration Ratio is the ratio of multi-day visitors in relation to the total visitors times the average stay in days (length of the event). Hotel Duration Ratio is a good number to measure different sized events as it takes into account the number of days after the event. Hotel Duration Ratio takes away any advantage or disadvantage in size in determining attractiveness of events. Using only Hotel Duration Ratio times the average stay can give lead to an estimate of the total occupancy in the motels. Using the simple formulas given the total estimate of the revenue can be found.

The last part of the rubric (Fig. 11) is the Event Gravitational Force Model. The Gravitational Force model numbers feed directly into both direct benefits and qualitative benefits such as branding and marketing of a venue or community. What the model provides that has not been available before is a clear graphical representation of how far out and how many participants attend certain events or attractions. In addition the model can be used to compute the average distance per attendee, which indirectly over time may show qualitative marketing and branding effects. Finally, Gravitational Force diagrams for significantly different events can be compared sideby-side or even on top of each other to get direct visual comparisons.

Rubric limitations and strategic considerations: 
The foregoing rubric is limited by the accuracy of estimates of visitor spending and by the limitations inherent in the Events Gravitational Force Model that have already been explained. Comparing events or attractions of different sizes is somewhat problematic in that the colored spokes shown in the gravity graphs do not clearly show the size differences in events being compared graphically. Decision makers will need to take size considerations into their thought processes. The ratios and formulas provided in this study help to numerically evaluate events beyond what the graphs show. Obviously, too, getting zip codes from visitors is essential to this kind of evaluation. Actual costs for community events are often elusive, as some events require extensive use of volunteers, local organizations and hidden costs for the use of multiple public agencies for policing, ambulance and other costs.

Strategically speaking, the authors would humbly suggest that events be first evaluated in terms of organizational priorities (such as cost, sustainability, profitability, local historical significance, legacy, branding and the like). Once the priorities are set then the Gravitational Force would be used to show graphically the patterns of demand for the event, how the event compares to other events and to help with the calculations of the direct benefits (as in Table 3). Additionally, over time the provided model may provide insight into how well the event, attraction, or the larger community is being marketed and branded.

Direct and indirect benefits: Direct benefits are quantitative and can be estimated, but some of the numbers are tricky due to variable tax rates, economic multipliers and lack of dependable data. Finding numbers and models that can accurately compare different events and venues is the central issue of this study. The ratios and measures the authors have settled on are shown in Table 3. The Hotel Ratio is the number of multi-day visitors divided by the number of day only visitors. The Hotel Ratio is higher when an event attracts a larger number of people who stay in hotels. The Hotel Duration Ratio is the number of multi-day visitors' times the average number of days that attendees stay at the event, then that number is divided by the total of all attendees. The Hotel Duration Ratio measures the propensity of attendees to stay in the community or the average stay for attendees. Multiplying the Hotel Duration Ratio by the estimated spending of people that stay overnight gives the estimated range of direct spending in the community and from that number the direct tax benefit can be estimated. None of these numbers include any multiplier effect, so the longterm economic effect will be higher.

All the numbers are helpful; however, the Hotel Ratio and Hotel Duration Ratio seem to be the most informative. The Hotel Duration Ratio is especially helpful as it leads directly to the calculations for the total revenues and the tax revenues. Indirect benefits such as community/event/venue branding are qualitative and hard to measure. But, over successive years the Event Gravity numbers, the Hotel Ratio and Hotel Duration Ratio, along with some of the other direct measures can indicate changes in branding and marketing effectiveness.

Establish priorities: After events and/or venues have been evaluated using the Gravitational Model and the direct benefits have been calculated, the next step is for the governing entity to establish priorities.
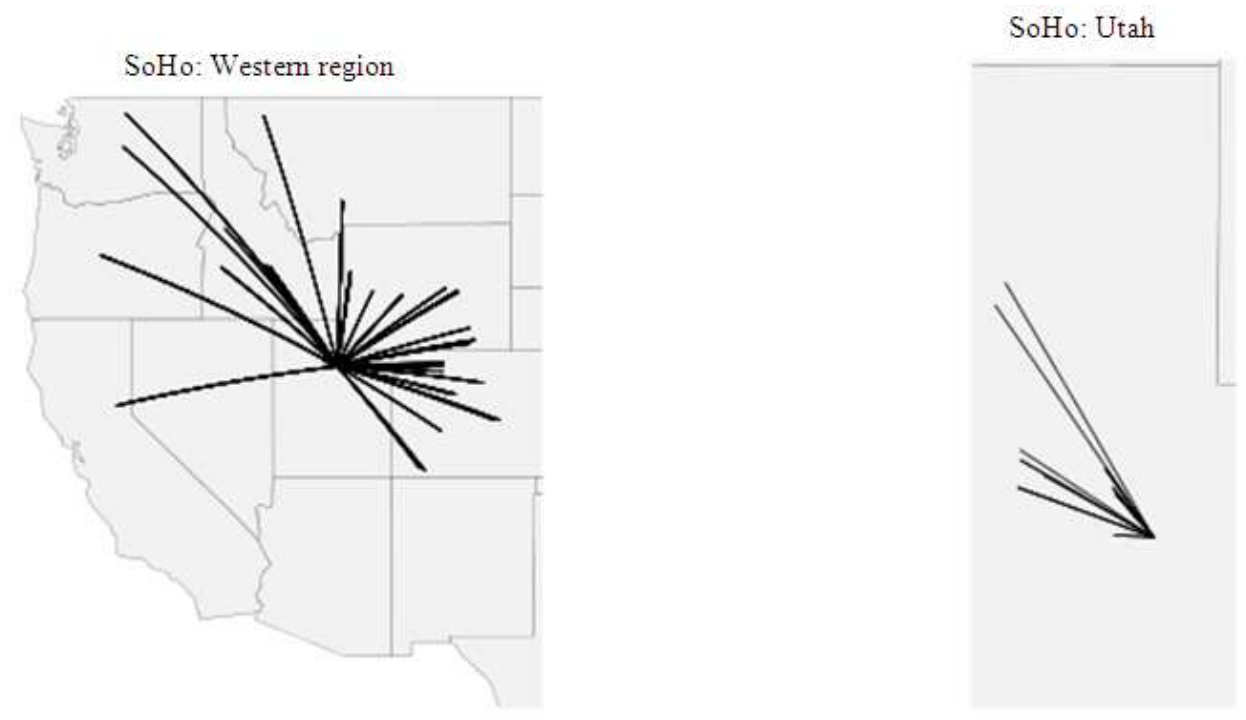

Fig. 10: Great Circle Histogram, SOHO, Magnified 

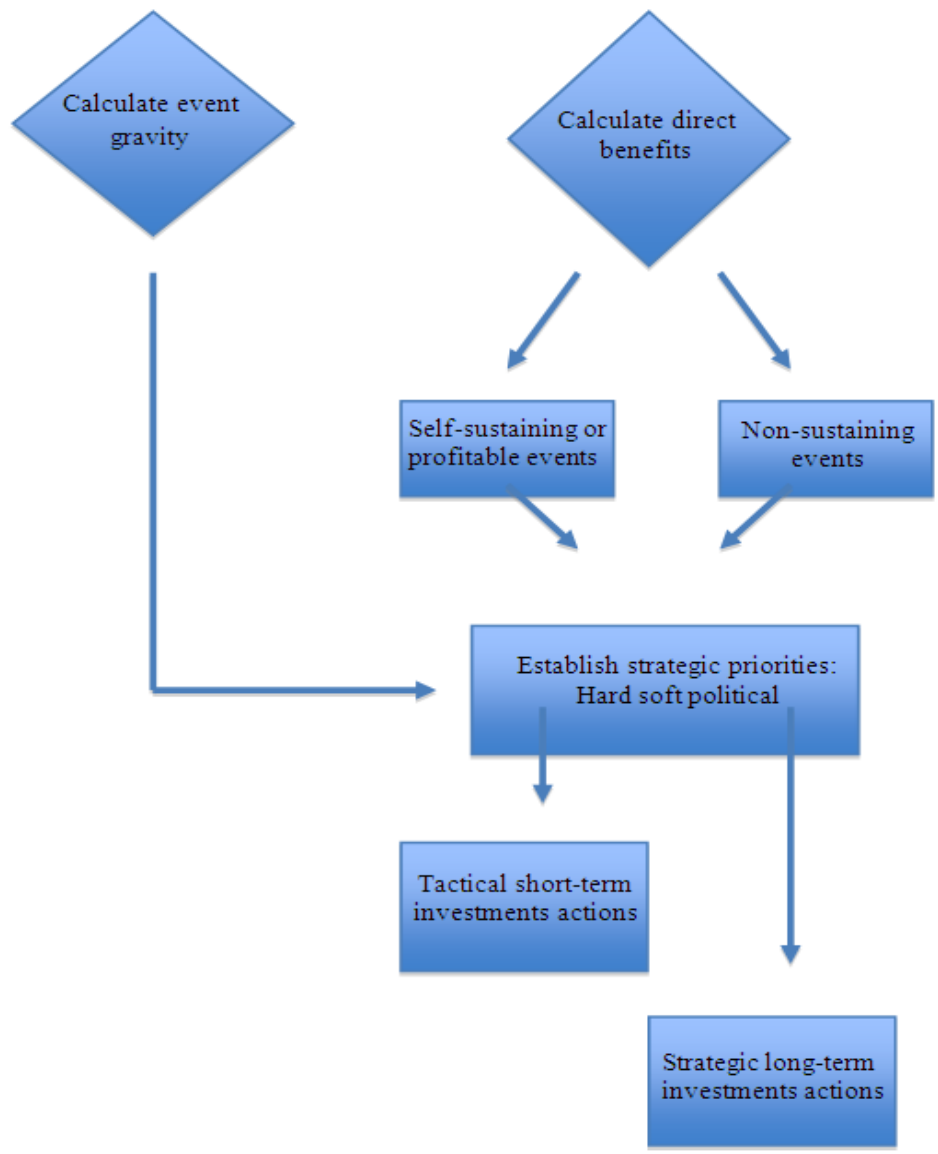

Fig. 11: Events and Decision Making Rubric

Table 3: Comparative ratios and economic measures

\begin{tabular}{lll}
\hline Formula/Ratio/Measure & Soldier Hollow JOs & Heber Valley RR \\
\hline Hotel ratio & $\approx 12.8$ & $<0.14$ \\
Hotel Duration ratio & $\approx 4.36$ & $\approx 0.12$ \\
Est. Total revenue range & $\$ 302,000-\$ 501,000$ & $\$ 1,300,000 /$ yr \\
Est. Per day revenue & $\$ 50,333-\$ 83,550$ & $\$ 5,200$ \\
Est. County tax rev. & $\$ 30,200-\$ 50,000$ & $\$ 0$ (sales tax exempt) \\
Incremental County Costs & - None- & Unknown \\
Venue Revenue <Costs> & $<\$ 22,023.63>(2010-11)$ & $\$ 1,300,000$ \\
& $<\$ 86,750.00>(2011-12)$ & \\
\hline
\end{tabular}

Note: Both the HVRR and Soldier Hollow are sales tax exempt so the only sales tax revenue from the two entities would come from the local economic multiplier; meaning, revenues staying in the community and being re-spent locally

Often this step is done first and leads to bad decision making as politics and other pressures override reality. The authors have identified three categories of priorities (hard, soft and political) and the order in which they are presented is not intended to be in order of importance. In different situations any of the three could be the most important and the third, political, is often a combination of both hard and soft priorities. In brief the three priorities are as follows:

- Hard: Economic value, Tax revenues, Public/Private costs, Community welfare

- Soft: Community cohesion, the Community is branding, Community heritage/history, Community emotional attachment

- Political: Voter preferences plus any combination of the Hard and Soft priorities

\section{CONCLUSION}

The Event Gravitational Force Model is shown in this study (Fig. 11) provides a visual and quantitative evaluation tool for events of all sizes for communities and organizations. The model measures and graphs attendees in relation to distance traveled and thus visually and numerically evaluates both direct 
benefits and branding/marketing over the long-term. Additionally, average distance traveled per attendee can be computed. Once the data is loaded into the model graphic and numeric comparisons between various competing events and attractions can be done side-by-side or transposed on the same graph.

Then events can be compared to each other using a cost benefit analysis using the Hotel Ratio and the Hotel Duration Ratio, with other costs and revenue considerations. The Hotel Ratio has shown the most dramatic results of the cost benefit analysis. The Hotel Ratio looks at the ratios of multi-day visitors to single day visitors, the higher the ratio the more money the community will bring in. With more information, multipliers, average night stay at the hotel; cost of meals, a full picture of potential revenue can be shown to decision makers on where to spend an ever decreasing pool of public funds.

The purpose of the study was to demonstrate, in a rough theoretical way, how the tools presently developed can be used to solve a problem many city, country, even state organizations are facing; which events to fund and which events to not fund. By examining where people come from four events, the number of people who stay longer than one day and the potential revenue from taxes, these organizations can better understand where to put the scarce money they have to get the maximum potential return for their communities.

\section{REFERENCES}

Adams, N.L. and L.L. Adams, 2006. Heber valley railroad marketing survey. Proceedings of the 4th Mountain Plains Management Conference, Orem, UT Quarter/Autumn.

Becker, R.A., A.R. Wilks, R. Brownrigg and T.P. Minka, 2011. Maps: Draw geographical maps.

Halladay, D. and R. Resnick, 1974. Fundamentals of Physics, Revised Printing. 1st Edn., John Wiley and Sons Inc., New York.

Hijmans, R.J., E. Williams and C. Vennes, 2011. Geosphere: Spherical Trigonometry.

Lamigueiro, O.P., 2011. "Great circles, raster, sp and lattice", Personal Blog.

Team, R.D.C., 2011. R: A Language and Environment for Statistical Computing. Vienna. 